\title{
Language before Thought
}

\author{
José Manuel Igoa
}

In response to "Just a Thought" (Vol. 5, No. 2).

To the editors:

When I began to plan a response to David Lobina's review of Inner Speech: New Voices, I had a number of different, rather unruly, experiences. A few blurred impressions and images started to take shape, and words and traces of incomplete sentences hovered in my mind, though I can't make out which came first. While I struggled to order these scattered pieces of thought, I pictured myself writing the opening sentence of this short essay, though it certainly did not resemble what I have written as its actual beginning. One could very well acknowledge that these thoughts were pieces of inner speech, not intended to be communicated to a reader, but expressive of my state of mind at that moment.

Lobina begins his review with a more dignified example of inner speech, taken from the novel Ulysses by James Joyce, a common source of examples of this phenomenon. He asks the same appropriate questions found in the literature on inner speech: Are these pieces of interior monologue a direct reflection of the thoughts of the character in the novel? Or are they merely an inwardly directed expression of his thoughts, but different from them? Similarly relevant questions are bound to follow. If inner speech episodes (ISEs) are inevitably couched in words, or some other language-like medium, would one particular thought be altered by changing one of its words or parts? And more importantly, to what extent does inner speech mirror the units, structure, and functions of overt speech? Do inner and overt speech share all their properties except articulation? Are all ISEs composed of words, phrases, and sentences, or can there be wordless ISEs? And one last but no less important question: Is inner monologue a universal phenomenon, or are there individual or cultural differences in the frequency of its use or the variety of its forms?

Inner Speech: New Voices addresses most of these questions through the contrasting theoretical positions and empirical interests of its chapter authors. Four major issues are covered in the book, which may be labeled as follows.
First is the ontology of inner speech: Is inner speech a form of thought, perhaps among others, or is it just a means of expression of otherwise unconscious thinking and reasoning processes? Second is the structure and components of inner speech. The book addresses the attendant questions of whether the components of inner speech form a unified whole or co-occur as separate parts, and if separate parts, then what properties each part exhibits, including its relevance, its representational character, and its ability to be assessed for accuracy. Third is the epistemology of inner speech, with its irreducible first-person phenomenology - that is, the fact that it is a privately experienced phenomenon. Its very privacy leads to the subsequent difficulty of undertaking its study with objective scientific methods. The fourth issue is the functions and uses of inner speech. This topic has spawned some controversy, since inner speech has been claimed to serve quite heterogeneous purposes. These range from comparatively local and time-bound uses, such as mental rehearsal as a memory aid, or monitoring the speaker's intentions and speech plans in language production, to more general and far-reaching cognitive functions. Examples include language acquisition and metacognitive abilities like self-regulation and executive control, including planning future courses of action, inhibiting inappropriate thoughts or responses, and solving problems. Finally, inner speech makes a critical contribution to second-order dynamics of thinking-that is, the uniquely human ability to reflect on one's own thoughts.

It is not my purpose to summarize and comment on the claims and arguments put forward in the book. Aside from Lobina's, there are other thoughtful reviews available in the philosophical literature. ${ }^{1}$ My major aims in the remainder of this letter are to assess and occasionally sharpen Lobina's points of criticism. Adopting a more personal take on the issues under discussion, I bring up a few topics related to inner speech that are largely overlooked in this book and elsewhere. Their exploration would help to clarify and enrich the study of this interesting psychological phenomenon.

Lobina's criticism of the book under review is not particularly incisive. But he makes some remarks in a critical 
spirit. Perhaps the most salient of these is his complaint that linguists are noticeably absent among the contributors to the volume. He points out a few topics of study where the appeal to linguistic knowledge would provide theoretical underpinnings to the research on inner speech. One particular area that Lobina raises in this regard is the role of syntax in the organization of linguistic utterances. Studies of inner speech are usually silent on this matter. They regularly focus on the other two major components of language, sound and meaning. It is plain that syntax is a necessary bridge to integrate sound and meaning in language, so it makes sense to inquire into what the syntactic structure of inner speech utterances might look like. Cursory descriptions that appear in the work of Lev Vygotsky in the mid-1930s, ${ }^{2}$ along with subsequent works by other authors, ${ }^{3}$ only suggest that ISEs exhibit an abbreviated or simplified form, and point out that inner speech strings tend to delete sentence subjects or topics in favor of predicates. The condensation of inner speech purportedly affects the phonological content of ISEs as well, but no detailed description of the structural aspects of inner monologues appears to be available regarding the grammar of sounds or of words and phrases. The focus is instead set on the meaning component, which Vygotsky and his contemporaries, as well as many present-day scholars, claim is the level of processing at which ISEs become available to consciousness. But how can the meaning of a mental sentence be consciously accessed without its form, be it phonological, orthographic, or visual? Moreover, what is the representational format in which this formless meaning is couched? The notion of pure meaning in isolation is an abstraction with no anchoring point to be entertained by bearers of mental states.

The problem of meaning in inner speech raises the question of whether the meaning of a sentence of inner speech is fully determined in a propositional format. Most researchers of inner speech seem to favor the view that inner speech episodes do have a propositional format, but some argue that the meaning of inner speech straddles the propositional level of representation so as to include context-sensitive information that is often necessary to disambiguate the bare propositional content of linguistic utterances. This discussion is reminiscent of the confrontation between minimalist and contextualist approaches to meaning in natural language regarding the purported semantic indeterminacy of sentences. ${ }^{4}$ Lobina seems to endorse a minimalist stance on this issue, whereby the propositional meaning of a sentence, what is said by the speaker, is established before the sentence is uttered, even to himself, as would be the case of inner speech. Insofar as inner speech is a suitable means to represent and support self-knowledge, it should also incorporate extralinguistic information, at the very least concerning the implicit speaker's intention. The point of contention in this regard is whether this extra piece of information is actually extralinguistic or, alternatively, is realized as a form of conversation with oneself, as some authors argue. ${ }^{5}$ This view aligns with Vygotsky's belief that inner speech is the internalization of conversation and therefore has a social origin.

Another concern voiced in Lobina's review is the familiar skepticism about the possibility of accomplishing a scientific study of inner speech given its private character and its over-reliance on conscious reports, however carefully controlled, as in the Descriptive Experience Sampling method. ${ }^{6}$ This shortcoming is not specific to this particular research topic in cognitive science. Studies of sentence understanding and production suffer a similar, though perhaps more attenuated, drawback. Each lacks an accurate specification of the output, in the case of comprehension processes, or input representations, in the case of production processes, for these cognitive operations. Similarly, neuroscientific methods are often believed to bear the stamp of scientific legitimacy in the study of cognition, but such evidence is no less indirect and based on conventional premises.

Beyond these methodological misgivings, Lobina concludes that speech is not a primary causal factor in thinking and reasoning processes. He bases his conclusion on theoretical grounds and on his own review of empirical evidence. In this way, he apparently sides with those who classify language as a subsidiary role in cognition, a medium of expression at best. This stance seems to be held by a minority of the contributors to the volume under review. ${ }^{7}$ One deserved criticism Lobina raises and develops in endnote 22 of his review concerns the ambiguous, if not downright mistaken, notion of language that is commonly used in the literature on inner speech, especially with regard to the generation of meaning in linguistic utterances. Here is where syntax comes into play. According to generative linguistic theory, which is the framework that Lobina has in mind, it is the underlying structure of sentences, and not their visible form, that is responsible for the fixation of meaning. Syntactic structure is inaccessible to consciousness, especially the bare syntactic structure that characterizes the Chomskyan notion of I-language. ${ }^{8}$ Of course, one need not support this view of syntax as a minimal structure devoid of morphological markers. Be that as it may, I think that any serious hypothesis supporting the linguistic status of thoughts ought to be more precise on the proper definition of language.

It is nonetheless true that Noam Chomsky brought some confusion to the characterization of his I-language in his attempt to underwrite the prevalent role of language in thinking and downplay its contribution to interpersonal communication. ${ }^{9}$ In stark contrast to his definition of I-language as an abstract system designed for syntactic computation, he has occasionally declared that language is a system for expressing thought in the form of inner speech. He thus brought it quite close to the Vygotskian 
conception of language as an instrument for self-regulation. This is all the more surprising, for inner speech is anything but an abstract system of rules. It is rife with irregularities, ungrammatical strings, deleted constituents, and other anomalies. If language is to be seen as an instrument for the expression of thought, talking to oneself could be viewed as an impoverished and distorted reflection of the complex structure and functioning of human reasoning. ${ }^{10}$

To close this letter, let me add "just a thought" about one particular trait of inner speech that is most cumbersome to me, namely, the extremely varied forms of manifestation that fall under the same label. Inner speech has been described under many different guises. Some authors emphasize its sensory-like, acoustic, phonological, or motor qualities, while others underscore its semantic or even pragmatic features. And there are still those who argue that other non-representational cognitive states, generally labeled as unsymbolized thinking, may also qualify as instances of ISEs. ${ }^{11}$ Such states have been described as "thinking a particular, definite thought without the awareness of that thought's being conveyed in words, images, or any other symbols." ${ }^{\prime 2}$ This idea has recently been voiced in reports on Twitter and YouTube and has hit popular news websites..$^{13}$ The diversity of guises may raise skepticism in scientific quarters, and runs the risk of rendering meaningless the very notion of inner speech. So perhaps the most convenient way to approach this intriguing experience is to avoid the temptation to fit it into a single mold. Treat it instead as a multifaceted phenomenon that calls for a joint contribution of different disciplines and approaches, including linguistics, of course.

\section{José Manuel Igoa}

José Manuel Igoa is Professor of Psychology at the Universidad Autónoma de Madrid.

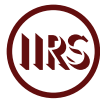

1. Two recent reviews of the book that I recommend are Marta Jorba, "Review of Inner Speech. New Voices (OUP), edited by Peter Langland-Hassan and Agustín Vicente," Notre Dame Philosophical Reviews (2019); and Daniel Gregory, “Inner Speech: New Voices," Analysis 80, no. 1 (2020): 16473, doi:10.1093/analys/anz096.

2. Lev Vygotsky, Thought and Language, trans. Eugenia Hanfmann and Gertrude Vakar (Cambridge: MIT Press, 1962). A more recent English translation by Alex Kozulin was published by MIT Press in 1986.

3. The condensed character of inner speech is highlighted in contemporary research as one of its varieties, alongside an expanded, dialogical form that retains the turn-taking qualities of conversation. See, for instance, Charles
Fernyhough, "Alien Voices and Inner Dialogue: Towards a Developmental Account of Auditory Verbal Hallucinations," New Ideas in Psychology 22 (2004): 49-68, doi:10.1016/j. newideapsych.2004.09.001. But some of the chapters in Inner Speech: New Voices report no empirical evidence of the condensed variety. See Russell Hurlburt and Christopher Heavey, "Inner Speaking as Pristine Inner Experience," in Inner Speech, 168-97. Other studies find it to be relatively uncommon when compared with the dialogical type. E.g., Ben Alderson-Day and Fernyhough, "Inner Speech: Development, Cognitive Functions, Phenomenology, and Neurobiology," Psychological Bulletin 141, no. 5, (2015): 93165, doi:10.1037/bul0000021).

4. According to semantic minimalism, sentences have a propositional content that is truth-evaluable and independent of contextual factors, except for indexical expressions-pronouns, demonstratives, time and space adverbials, and so forth-whose referents are contextually specified. This view is expressed in Emma Borg, Minimal Semantics (Oxford: Oxford University Press, 2004); and in Herman Cappelen and Ernest Lepore, Insensitive Semantics (Oxford: Blackwell, 2005). Contextualism, on the other hand, claims that the propositional contribution of an expression is not fully determined by the invariant meaning conventionally associated with the expression type, but depends upon the context. See Robyn Carston, Thoughts and Utterances: The Pragmatics of Explicit Communication (Oxford: Blackwell, 2002); and François Recanati, Literal Meaning (Cambridge: Cambridge University Press, 2004).

5. Outright advocates of the conversational character of inner speech in the book are Christopher Gauker, "Inner Speech as the Internalization of Outer Speech," 53-77; Keith Frankish, "Inner Speech and Outer Thought," 221-42; and Alain Morin, "The Self-Reflective Functions of Inner Speech," 276-98.

6. For a detailed description of this method, see Hurlburt and Heavey, "Inner Speaking as Pristine Inner Experience," Inner Speech: New Voices, 168-97.

7. The view of inner speech as an expression of thought is endorsed in Sam Wilkinson and Fernyhough's chapter, "When Inner Speech Misleads," 244-60; and in Edouard Machery's chapter, "Know Thyself: Beliefs vs. Desires in Inner Speech," 261-75.

8. A contrast is drawn between "external" language, or E-language, and I-language, where the "I" stands for internal, individual, implicit. This dichotomy was first proposed by Noam Chomsky in Knowledge of Language: Its Nature, Origin, and Use (New York: Praeger, 1986), and was later endorsed many times. The dichotomy loosely corresponds to the earlier distinction made by Chomsky between competence (akin to I-language), which is the body of implicit knowledge of grammar possessed by every speaker-hearer of a natural language, and performance (related to E-language), which is the real-time use of this internal body knowledge for various purposes. In the most recent devel- 
opment of generative theory, the Minimalist Program, Chomsky has reduced the contents of I-language to a single rule or operation called "Merge" that creates hierarchical structures by recursively combining pairs of lexical units to form larger sets. The results of this operation are broadcast to two external systems, one involved in semantic interpretation, and the other engaged in externalization for communicative purposes.

9. See the article by Norbert Wiley, "Chomsky's Anomaly: Inner Speech," International Journal for Dialogical Science 8, no. 1 (2014): 1-11, where the author gives examples of Chomsky's statements presenting inner speech as the medium of thought, and analyzes the contradictions and anomalies Chomsky falls into by following this path.

10. Within generative linguistics, it has been claimed that human thought acquires a systematic, formal semantics from grammar, particularly from the apparatus of Merge and its way of operating. The working hypothesis underlying this proposal is that the computational system of language enables the alignment of syntax and semantics, such that semantic structures come to mirror syntactic configurations of language. See Wolfram Hinzen, "Language and Thought," in The Oxford Handbook of Linguistic Minimalism, ed. Cedric Boeckx (Oxford: Oxford University Press, 2011). However, inner speech as it is commonly understood has no place in this picture of the linguistic embodiment of thought.
11. The original proponents of the construct of "unsymbolized thinking” were Russell Hurlburt and Christopher Heavey in their book Exploring Inner Experience (Amsterdam: John Benjamins, 2006). Subsequent criticisms and restatements of this controversial construct can be found in Agustín Vicente and Fernando Martínez-Manrique, "The Nature of Unsymbolized Thinking," Philosophical Explorations 19, no. 2 (2016): 173-87, doi:10.1080/13869795.2016.1176234; and Daniel Gregory, "Commentary: The Nature of Unsymbolized Thinking," Frontiers in Psychology 9, no. 216 (2018), doi:10.3389/fpsyg.2018.00216.

12. Quotation from Russell Hurlburt and Christopher Heavey, "The Phenomena of Inner Experience," Consciousness and Cognition 17 (2008): 798-810, doi:10.1016/j. concog.2007.12.006, cited in Vicente and Martínez-Manrique, "The Nature of Unsymbolized Thinking," 174.

13. I have selected three representative examples of testimonies from different people concerning their inner speech experiences or lack thereof. The first one is a YouTube video by Ryan Langdon entitled "Q\&A with a Person Who Does Not Have an Internal Monologue," YouTube video, uploaded January 31, 2020. The second and third examples come from online print media: Melissa Malamut, "Scientists Explain the Viral 'Internal Narrative' Phenomenon," New York Post, February 5, 2020; Amanda Cassidy, "Can You Actually Hear Your Inner Voice? The Internet Is Split," Image Magazine, February 1, 2020. 